\title{
Trigeminal sensitivity in chronic rhinosinusitis: topographical differences and the effect of surgery*
}

\section{Sophia C. Poletti”, Mandy Cuevas\#, Sina Weile, Thomas Hummel}

Smell and Taste Clinic, Department of Otorhinolaryngology, TU Dresden, Dresden, Germany
Rhinology 55: 70-74, 2017

https:://doi.org/10.4193/Rhino16.194

*Received for publication:

June 15, 2016

Accepted: September 19, 2016

\# Shared first authorship

\begin{abstract}
Introduction: Little is known on endonasal trigeminal sensitivity in patients with chronic rhinosinusitis with nasal polyps (CRSwNP). The aim of our study was to investigate changes in trigeminal sensitivity in patients with CRSwNP and the effect of functional endoscopic sinus surgery (FESS) on trigeminal perception.
\end{abstract}

Methods: A prospective study was performed to investigate the trigeminal sensitivity at three different locations within the nose (anterior septum, anterior lateral wall, middle turbinate) using electrical stimuli. Therefore 45 CRSwNP patients were compared to 30 healthy subjects. Further, the effect of FESS was investigated in 31 patients before and 3 months after surgery.

Results: CRSwNP patients had a significantly higher trigeminal threshold at all tested locations than healthy subjects. The lowest trigeminal detection threshold could be shown at the "entrance" of the nose in healthy subjects and in patients with CRSwNP. Three months after FESS a significant improvement of trigeminal detection threshold was observed at the anterior nasal septum.

Conclusion: Protective function of the trigeminal system is preserved in CRSwNP patients. FESS seems to show bene cial effects on restoring sentinel function at the "entrance" of the nose.

Key words: nose, sinusitis, nasal surgical procedure, innervation, nasal mucosa

\section{Introduction}

Chronic rhinosinusitis with nasal polyps (CRSwNP) is reported to have a prevalence of $2.7 \%$ in the general population ${ }^{(1)}$. Apart from high costs ${ }^{(2)}$ associated with CRS there is a significant impact on patients' quality of life. Most commonly reported and apparently most bothersome symptom seems to be the sensation of nasal obstruction in around $90 \%{ }^{(3)}$ followed by nasal discharge, facial pain and olfactory dysfunction ${ }^{(4)}$.

The influence of endonasal trigeminal perception on the sensation of a "free nose" (5) but also on the feeling of nasal obstruction without changes in airflow resistance ${ }^{(6)}$ could be demonstrated earlier.

A more recent study demonstrated that not only air temperature but also air humidity affected the trigeminal sensation of nasal patency. Thus, increased patency perception could be achieved through mucosal heat loss/ cooling ${ }^{(7,8)}$. Further, a higher trige- minal detection threshold in patients undergoing septoplasty compared to healthy subjects revealed the important role of endonasal trigeminal sensitivity in patients with nasal breathing difficulties scheduled for surgery ${ }^{(9)}$.

In patients with chronic rhinosinusitis (CRS) where the sensation of nasal blockage is a cardinal symptom, a decreased trigeminal sensitivity could be demonstrated ${ }^{(10)}$. Whether mucosal inflammation in CRS contributed to changes in trigeminal perception is not entirely understood. But local inflammation after allergen exposure was associated with significant changes of trigeminal event- related potentials in patients with allergic rhinitis (11). The main aim of our study was to investigate changes in trigeminal sensitivity in patients with CRSwNP. Further, we aimed to investigate the effect of functional endoscopic sinus surgery on trigeminal perception, hypothesizing trigeminal regeneration 3 months after surgery. 


\section{Materials and methods}

\section{Participants}

A prospective study was performed at the tertiary centre of the Smell and Taste Clinic (Department of Otolaryngology) of the "Technische Universität" (TU) Dresden. The study was conducted according to the Declaration of Helsinki and approved by the Ethics Committee of the Medical Faculty of the TU Dresden (application number: EK20012013). All subjects and patients provided informed consent prior to inclusion into the study. Controls consisted of 30 healthy participants without any history of chronic rhinosinusitis or olfactory disorders. The case group included 45 patients with chronic rhinosinusitis with nasal polyps (CRSwNP). Participants were only included if their age ranged between 18 and 50 years. All patients underwent endoscopic sinus surgery for the first time which was an inclusion criterion for the case group. All tests were undertaken one day prior and 3 months after surgery. As only 31 out of 45 patients appeared for post operative examination, comparison between pre and post operative test results was done in those 31 patients. All study participants underwent a complete ENT examination including nasal endoscopy.

\section{Self- assessment of nasal breathing and olfactory ability} All study participants had to rate their nasal breathing and olfactory ability on a visual numeric scale ranging from " 1 - very good" to "7 - no nasal breathing/ no olfactory function".

\section{Lund-Kennedy-Score}

To evaluate the severity of CRSwNP all patients were graded according to the Lund-Kennedy-Score. Polyp size, mucosal oedema and secretion was evaluated using nasal endoscopy. Polyp size was scored as 0 - no polyps, 1 - polyps up to the middle turbinate, 2- polyps filling out nasal cavity. Mucosal oedema was rated as 0 - no oedema, 1 - mild/ moderate, 2 - polypoid change and secretion as 0 - no secretion, 1- hyaline, 2- purulent. The sum of each category was defined as the Lund-Kennedy-Score.

\section{Olfactory identification test}

The odor identification task ${ }^{(12)}$ was based on the identification of 16 common odors presented on felt- tip pen like odor dispensers. Odors were presented to the patient for 2 seconds about $2 \mathrm{~cm}$ in front of the nostril. Patients had to choose from a list of four descriptors in a forced choice procedure. Identifying 12 or more odors out of 16 was considered normosmic.

\section{Endonasal trigeminal detection threshold}

Trigeminal detection threshold was measured at three different locations: at the anterior nasal septum $(1 \mathrm{~cm}$ from the nasal vestibule), the anterior lateral nasal wall $(1 \mathrm{~cm})$ and the middle turbinate $(4.5 \mathrm{~cm})$. Electrical stimuli were applied through a spherical electrode (PowerLab ADInstruments, Spechbach,
Germany) which was placed under 30 endoscopic vision at above given locations. To avoid movements of the electrode it was fixed to a spectacle frame that was worn by each study participant. The stimulus duration was set at $50 \mu$ s. Starting with a stimulus intensity of $0.05 \mathrm{~mA}$, intensity was gradually increased by $0.05 \mathrm{~mA}$ until the subject detected the stimulus. Then the intensity was decreased by $0.05 \mathrm{~mA}$ steps till the subject could not detect the stimulus any more. From there again stimulus intensity was increased by $0.01 \mathrm{~mA}$ steps until the stimulus could be perceived again, which was then defined as the trigeminal detection threshold.

The trigeminal detection threshold was compared between the 3 endonasal locations in healthy subjects and in CRS patients. Further, comparison of threshold was done between healthy subjects and CRS patients before functional endoscopic sinus surgery and also between patients before and 3 months after FESS.

\section{"Air puff" testing}

Two high density polyethylene squeeze bottles $(250 \mathrm{ml})$ with a pop-up spout were placed into each nostril. By pressing both empty bottles at the same time using a hand-held squeezing device ${ }^{(13)}$, an air puff with similar volume was administered to both nostrils. Patients had to rate the intensity of the air puff in both nostrils separately on a visual numeric scale ranging from 0 - no sensation to 10 - very intense.

\section{Statistical analysis}

Data analyses were done using SPSS (Statistical Package for Social Sciences, version 23.0, SPSS Inc., Chicago, IL, USA). The level of significance was set at $p=0.05$. For group comparisons t-tests and $\mathrm{Chi}^{2}$ tests were applied. Psychophysical data were analysed using analyses of variance (ANOVA) for repeated measures ("within-subject-factor" [measurements pre- and post-operatively] and "between-subject-factor" [group]). Correlation analyses were performed according to Pearson.

\section{Results}

The control group consisted of 30 healthy subjects ( 15 men, 15 women) with an average age of 36 years ranging from 22 to 50 years (9.9 SD). Forty- five patients could be included in the case group with an average age of 37 years (range: 24 to 49 years, 8.3 SD; 15 women, 30 men). Five (17\%) control subjects were smokers whereas 18 (40\%) patients from the case group smoked regularly. There was no significant difference between the two groups concerning age $(p=0.83)$, gender $(p=0.15)$ or smoking habits $(p=0.06)$.

All of the 45 patients underwent endoscopic sinus surgery for chronic rhinosinusitis with nasal polyps whereas only 31 patients (20 men, 11 women, mean age 37 years) appeared for postoperative examination. To compare pre and postoperative trigemi- 
Table 1. Comparison of trigeminal detection threshold between different endonasal locations in healthy subjects and CRSwNP patients before surgery.

\begin{tabular}{|c|c|c|c|c|}
\hline & $\begin{array}{c}\text { Anterior } \\
\text { nasal } \\
\text { septum }\end{array}$ & $\begin{array}{l}\text { Middle } \\
\text { turbinate }\end{array}$ & $\begin{array}{l}\text { Anterior } \\
\text { lateral } \\
\text { nasal wall }\end{array}$ & p value \\
\hline \multirow[t]{2}{*}{$\begin{array}{l}\text { Subjects } \\
(n=30)\end{array}$} & $0.77 \pm 0.32$ & $1.05 \pm 0.50$ & & 0.01 \\
\hline & & $1.05 \pm 0.50$ & $0.8 \pm 0.34$ & 0.01 \\
\hline \multirow[t]{2}{*}{$\begin{array}{l}\text { Patients } \\
(n=45)\end{array}$} & $1.03 \pm 0.46$ & $1.34 \pm 0.59$ & & $<0.001$ \\
\hline & & $1.34 \pm 0.59$ & $0.9 \pm 0.36$ & $<0.001$ \\
\hline
\end{tabular}

nal perception those 31 patients only were taken into account. Out of those 31 patients 7 received before and 14 after surgery nasal topical steroid treatment. Further, 11 out of 31 patients received at least once systemic steroids during the course of their disease before surgery.

\section{Self-assessment of nasal breathing and olfactory ability}

There was a significant difference in self rating of the nasal breathing ability between the two groups (right side: patients: $4.8 \pm 1.5$ SD, subjects: $3.3 \pm 1.6$ SD, $p<0.001$; left side: patients: $5.0 \pm 1.5$ SD, subjects: $3.4 \pm 1.5 \mathrm{SD}, \mathrm{p}<0.001$ ) and self rating of the olfactory ability (patients: $4.8 \pm 1.7 \mathrm{SD}$, subjects: $3.4 \pm 1.6 \mathrm{SD}$, $p=0.001)$.

\section{Endonasal trigeminal perception threshold}

In healthy subjects as well as in pre operative CRSwNP patients a significant difference between the trigeminal detection threshold at the nasal septum and the middle turbinate and between the lateral nasal wall and the middle turbinate could be shown, indicating that both groups were most sensitive at the septum and the lateral nasal wall (Table 1).

Comparing healthy subjects with CRSwNP patients before sinus surgery we could demonstrate a significantly higher trigeminal perception threshold (=lower sensitivity) at all three locations in CRSwNP patients (Table 2).

Comparing pre and post operative CRSwNP patients $(n=31) 3$ months after endoscopic sinus surgery we could present a significant improvement of trigeminal sensitivity at the nasal septum in post operative patients. No significant difference could be shown at the lateral nasal wall or the middle turbinate (Table 3). Regarding topical steroid treatment, no significant differences ( $p s>0.05$ ) were found between treated and non treated patients in detection threshold changes postoperatively at all three locations. Likewise, no significant differences in trigeminal detection threshold at all locations appeared between systemi-
Table 2. Comparison of trigeminal detection threshold between healthy subjects and CRSwNP patients before surgery.

\begin{tabular}{|c|c|c|c|}
\hline & $\begin{array}{l}\text { Subjects } \\
(n=30)\end{array}$ & $\begin{array}{l}\text { Patients } \\
(n=45)\end{array}$ & $p$ value \\
\hline $\begin{array}{l}\text { Anterior nasal } \\
\text { septum }\end{array}$ & $0.77 \pm 0.32$ & $1.03 \pm 0.46$ & 0.03 \\
\hline $\begin{array}{c}\text { Middle } \\
\text { turbinate }\end{array}$ & $1.05 \pm 0.5$ & $1.34 \pm 0.59$ & 0.03 \\
\hline $\begin{array}{c}\text { Anterior lateral } \\
\text { nasal wall }\end{array}$ & $0.8 \pm 0.34$ & $0.9 \pm 0.36$ & 0.04 \\
\hline
\end{tabular}

cally treated and non- treated patients (ps > 0.05).

\section{Lund-Kennedy-Score}

As the control group consisted of healthy subjects without nasal polyps all subjects had a score of 0 . A significant difference between the pre and postoperative scores of 31 patients undergoing endoscopic sinus surgery could be shown (pre: score 4.9, 1.4 SD; post: score 0.6, 1.2 SD; $p<0.001$ ). There was no significant correlation between the Lund-Kennedy-Score and the trigeminal perception threshold at the nasal septum $(p=0.60)$, the middle turbinate $(p=0.52)$ or the lateral nasal wall $(p=0.22)$.

\section{Olfactory identification test}

Control subjects had an average score of 13.9 (1.06 SD) points on identification testing whereas patients reached on average 11.4 (5.0 SD) points only. Healthy subjects performed significantly better on the odor identification task than patients pre operatively $(p=0.01)$. Comparison between pre and post operative results revealed no significant difference (pre: score 11.8, 4.5 SD; post: score 13, 3.2 SD; $p=0.14$ ).

\section{"Air puff" testing}

No significant difference in air puff rating between healthy subjects and patients (subjects: $1.05 \pm 0.48 \mathrm{SD}$; patients: $1.0 \pm 1.22$ $\mathrm{SD}, \mathrm{p}=0.64$ ) or between pre and postoperative patients (pre: 1.0 \pm 1.47 SD; post: $2.0 \pm 1.75 \mathrm{SD}, \mathrm{p}=0.12$ ) could be shown. Further, no correlation between the air puff rating and the trigeminal perception threshold at the nasal septum $(p=0.92)$, the middle turbinate $(p=0.43)$ or the anterior lateral nasal wall $(p=0.68)$ was observed.

\section{Discussion}

Major results found in this study include: 1) the demonstration of the lowest trigeminal detection threshold at the "entrance" of the nose in healthy subjects (anterior nasal septum), 2) as well as 
Table 3. Comparison of trigeminal detection threshold in CRSwNP patients before and three months after surgery.

\begin{tabular}{|c|c|c|c|}
\hline & $\begin{array}{c}\text { Pre operative } \\
\qquad(n=31)\end{array}$ & $\begin{array}{l}\text { Post operative } \\
\qquad(n=31)\end{array}$ & p value \\
\hline $\begin{array}{l}\text { Anterior nasal } \\
\text { septum }\end{array}$ & $1.01 \pm 0.41$ & $0.85 \pm 0.07$ & 0.05 \\
\hline $\begin{array}{l}\text { Middle } \\
\text { turbinate }\end{array}$ & $1.33 \pm 0.6$ & $1.16 \pm 0.49$ & 0.12 \\
\hline $\begin{array}{c}\text { Anterior lateral } \\
\text { nasal wall }\end{array}$ & $0.86 \pm 0.26$ & $0.87 \pm 0.28$ & 0.92 \\
\hline
\end{tabular}

in CRSwNP patients (anterior lateral nasal wall); 3) CRSwNP patients had a significantly higher trigeminal detection threshold at all tested locations (the anterior nasal septum, the anterior lateral nasal wall and the middle turbinate) than healthy subjects; 4) 3 months after functional endoscopic sinus surgery a significant improvement of trigeminal perception was observed at the anterior nasal septum.

Several methods with regard to stimulus site and quality have been used in the past to assess endonasal trigeminal sensitivity. All studies showed more or less consistently highest chemosensory ${ }^{(14,15)}$ but also somatosensory ${ }^{(16)}$ sensitivity at the anterior part of the nose. As the anterior nasal mucosa is the region where inhaled airstream first hits the mucosa, highest trigeminal sensitivity in this location is thought to have a sentinel function to protect the airway from potentially harmful agents. In line with these findings, a similar distribution of trigeminal sensitivity was found in CRSwNP patients albeit at a lower level of sensitivity. This indicates that the chronic inflammatory process seems to generally affect the intranasal trigeminal system while the sentinel function is tried to be maintained. Considering the sensation of nasal patency, a significantly lower trigeminal sensitivity in CRSwNP patients might contribute additionally to mechanical obstruction through nasal polyps to the sensation of nasal obstruction. To our knowledge this is the first study to present topographical differences of somatosensory sensitivity in CRS patients.

The influence of inflammatory mediators on various trigeminal receptors within the nasal mucosa ${ }^{(17,18)}$ could be demonstrated earlier. Also changes in trigeminal response to allergen exposure of allergic rhinitis patients suggested a meaningful impact of inflammation on trigeminal perception ${ }^{(11)}$. Whether inflammation induces an increase or decrease in trigeminal sensitivity also seems to depend on the duration of inflammation (acute or chronic) ${ }^{(19)}$. Similar to those findings, a decrease in trigeminal sensitivity could be presented in CRSwNP patients, where endonasal inflammation per definition lasted for more than 3 months.

The interaction between the olfactory and trigeminal system is well documented showing decreased trigeminal sensitivity in dysosmic patients ${ }^{(13,20-22)}$. Olfactory dysfunction is amongst the most frequent symptoms in CRS patients with a prevalence of up to $90 \%{ }^{(23)}$. In our study a significantly lower odor identification score confirmed olfactory impairment in CRS patients. With regard to those findings, an interactions between those two systems leading to lower trigeminal sensitivity in CRS patients could be taken into account. But at the same time, there is evidence that changes in trigeminal sensitivity seems to be specific for chemosensory rather than somatosensory sensation making this explanation questionable.

To our knowledge, this is the first time the effect of functional endoscopic sinus surgery on endonasal trigeminal sensitivity was investigated. Reducing inflammation by removing polyps, widening sinus drainage pathways and improving nasal/sinus ventilation seems to improve trigeminal perception, at least to some extent, in a so far unknown way. But as trigeminal regeneration 3 months after FESS was mainly seen at the anterior part but not at the posterior regions of the nose, it may be hypothesized that apart from mucosal inflammation compression of the nasal mucosa caused by polyps might have induced irrevocable damage to trigeminal nerve endings. Coming back to the interaction between the olfactory and trigeminal system, an improvement of trigeminal sensitivity could be observed along with the improvement of olfaction, at least in patients with post infectious dysosmias ${ }^{(21)}$. Whether restricted improvement of trigeminal sensitivity 3 months after FESS as shown in our patients related to unchanged olfactory abilities after FESS should be considered as well. However, our data shows that FESS is associated with an improvement of trigeminal sensitivity at the anterior domain of the nose which means that the protective function of the respiratory tract is maintained. This potentially beneficial effect of FESS on the trigeminal sensitivity could be of particular importance in patients with pulmonary affection like in aspirin- exacerbated respiratory disease (AERD) where the protection of a weakened lower respiratory system is crucial. And as the severity of polyposis in these patients is known to be associated with the frequent need of FESS ${ }^{(24)}$ a potentially protective effect of FESS might be of further advantage. However, the study design we used does not allow any statements on causality of sentinel function restoration.

Even though the effect of steroids on the trigeminal sensitivity was not in focus of our study our data indicates that steroids seem not to show any major effects on endonasal trigeminal detection threshold in CRSwNP patients. But further studies are needed to assess the influence of steroids on endonasal trigeminal sensitivity.

According to Meusel et al. ${ }^{(14)}$, a high responsiveness to menthol within the posterior regions suggested the presence of TRPM8 receptor ${ }^{(25)}$, a cold receptor most probably responsible for the perception of nasal patency, in those regions. In our study we 
could not show trigeminal detection threshold improvement within the posterior nasal regions using electrical stimuli. Electrical stimuli are thought to be unspecific and therefore seem to activate a wide range of receptors simultaneously and probably even axons directly. Hence, decreased trigeminal sensitivity could also indicate a lack of functional TRPM-8 receptors. In this case, if patients do not improve in terms of nasal breathing ability after FESS one should consider a lack of improvement in airstream detecting cold- receptors in the posterior domains of the nose. But further investigations are needed to assess distribution and functionality of TRPM-8 receptors in CRS patients.

\section{Acknowledgement}

This work was supported by a grant from the Deutsche Forschungsgemeinschaft to TH (DFG HU441/18-1). SCP was supported by the Dresden Junior Fellowship Programme of the "Technische Universität" (TU) Dresden.

\section{Authorship contribution}

TH: Contributed to study conception and design, analysis and interpretation of the data, critical revision of the manuscript. SW: Contributed to study conception, data acquisition and critical revision of the manuscript.

MC: Contributed to study conception, data acquisition, interpretation of the data and critical revision of the manuscript. SCP: Contributed to analysis and interpretation of the data, drafting and revising of the manuscript.

\section{Conflict of interest}

None of the authors reports a potential conflict of interest.

\section{References}

1. Johansson L, Akerlund A, Holmberg K Melén I, Bende M. Prevalence of nasal polyps in adults: the Skövde population-based study. Ann Otol Rhinol Laryngol. 2003 Jul;112(7):625-9.

2. Smith KA, Orlandi RR, Rudmik L. Cost of adult chronic rhinosinusitis: A systematic review. Laryngoscope 2015;125(7):1547-56.

3. Damm M, Quante G, Jungehuelsing $M$ Stennert E. Impact of functional endoscopic sinus surgery on symptoms and quality of life in chronic rhinosinusitis. Laryngoscope 2002 Feb;112(2):310-5.

4. Fokkens WJ, Lund VJ, Mullol J et al. European Position Paper on Rhinosinusitis and Nasal Polyps 2012. Rhinol Suppl. 2012 Mar;(23):1-298.

5. Eccles R, Jones AS. The effect of menthol on nasal resistance to air flow. J Laryngol Otol. 1983:97(8):705-9.

6. Jones AS, Crosher R, Wight RG, Lancer JM Beckingham E. The effect of local anaesthesia of the nasal vestibule on nasal sensation of airflow and nasal resistance. Clin Otolaryngol Allied Sci. 1987 Dec;12(6):4614.

7. Zhao K, Blacker K, Luo Y, Bryant B, Jiang J. Perceiving nasal patency through mucosal cooling rather than air temperature or nasal resistance. PLoS One 2011;6(10).

8. Zhao K, Jiang J, Blacker $\mathrm{K}$ et al. Regional peak mucosal cooling predicts the perception of nasal patency. Laryngoscope 2014;124(3):589-95.

9. Scheibe M, Schulze S, Mueller CA, Schuster $B$, Hummel T. Intranasal trigeminal sensitivity: measurements before and after nasal surgery. Eur Arch Otorhinolaryngol. 2014 Jan;271(1):87-92

10. Saliba J, Fnais N, Tomaszewski $M$ et al. The role of trigeminal function in the sensation of nasal obstruction in chronic rhinosinusitis. Laryngoscope 2016 May; 126(5):E174-8

11. Doerfler $H$, Hummel $T$, Klimek L, Kobal
G. Intranasal trigeminal sensitivity in subjects with allergic rhinitis. Eur Arch Otorhinolaryngol. 2006 Jan;263(1):86-90.

12. Hummel T, Sekinger B, Wolf SR, Pauli E, Kobal G. "Sniffin" sticks': olfactory performance assessed by the combined testing of odor identification, odor discrimination and olfactory threshold. Chem. Senses 1997 Mar;22(1):39-52.

13. Hummel T, Futschik T, Frasnelli J, Hüttenbrink KB. Effects of olfactory function, age, and gender on trigeminally mediated sensations: A study based on the lateralization of chemosensory stimuli. Toxicol Lett. 2003;140-141:273-80.

14. Meusel T, Negoias S, Scheibe M, Hummel T. Topographical differences in distribution and responsiveness of trigeminal sensitivity within the human nasal mucosa. Pain. International Association for the Study of Pain; 2010;151(2):516-21.

15. Frasnelli J, Heilmann S, Hummel T. Responsiveness of human nasal mucosa to trigeminal stimuli depends on the site of stimulation. Neurosci Lett. 2004;362(1):65-9.

16. Scheibe M, Schmidt A, Hummel T. Investigation of the topographical differences in somatosensory sensitivity of the human nasal mucosa. Rhinology 2012;50(3):290-3.

17. Linte RM, Ciobanu C, Reid G, Babes A. Desensitization of cold- and menthol-sensitive rat dorsal root ganglion neurones by inflammatory mediators. Exp Brain Res. 2007:178(1):89-98.

18. Taylor-Clark TE, Undem BJ, Macglashan DW, Ghatta S, Carr MJ, McAlexander MA. Prostaglandin-induced activation of nociceptive neurons via direct interaction with transient receptor potential A1 (TRPA1). Mol Pharmacol. 2008;73(2):274-81.

19. Benoliel R, Biron A, Quek SYP, Nahlieli O, Eliav E. Trigeminal neurosensory changes following acute and chronic paranasal sinusitis. Quintessence Int. 2006 Jun [cited
2016 May 13];37(6):437-43.

20. Hummel T, Barz S, Lötsch J, Roscher S, Kettenmann B, Kobal G. Loss of olfactory function leads to a decrease of trigeminal sensitivity. Chem Senses. 1996 Feb;21(1):759.

21. Frasnelli J, Schuster B, Hummel T. Olfactory dysfunction affects thresholds to trigeminal chemosensory sensations. Neurosci Lett. 2010 Jan 14;468(3):259-63.

22. Frasnelli J, Hummel T. Interactions between the chemical senses: trigeminal function in patients with olfactory loss. Int J Psychophysiol. 2007 Sep;65(3):177-81.

23. Litvack JR, Fong K, Mace J, James KE, Smith TL. Predictors of olfactory dysfunction in patients with chronic rhinosinusitis. Laryngoscope 2008;118(12):2225-30.

24. Kowalski ML. Aspirin-sensitive rhinosinusitis and asthma. Clin Allergy Immunol. 2007 Jan;19:147-75.

25. Peier AM, Moqrich A, Hergarden AC et al. A TRP channel that senses cold stimuli and menthol. Cell 2002;108(5):705-15.

Thomas Hummel, M.D.

Smell \& Taste Clinic

Department of Otorhinolaryngology

TU Dresden

Fetscherstrasse 74

01307 Dresden

Germany

Tel: +49-351-458-4189

Fax +49-351-458-4326

E-mail:

thummel@mail.zih.tu-dresden.de 